\title{
THEORETICAL ANALYSIS OF TURBULENT FLOW AND HEAT TRANSFER AROUND A SURFACE-MOUNTED OBSTACLE
}

\author{
TSUTOMU ARAGAKI, Shuichi IWATA, Hideto TANGE, \\ SETSURO HIRAOKA AND IKUHO YAMADA \\ Department of Applied Chemistry, Nagoya Institute of Technology, \\ Nagoya 466 \\ FUMIO KAWAIZUMI \\ Institute for Molecular Science, Okazaki 444
}

Key Words: Heat Transfer, Augmentation, Surface-Mounted Obstacle, Turbulent Flow, Separated Flow, Reattachment Length, Nusselt Number, Finite Element Method

\begin{abstract}
To estimate the distribution of turbulent eddy viscosity in the flow related to complicated geometries, a simple model was developed based on the mixing-length hypothesis. This model was applied to two cases of turbulent separated flows around a surface-mounted obstacle of circular cross section. The first case is film flow on the outer wall of a vertical tube, and the second is flow between parallel plates. Calculations were performed by using the Galerkin finite-element method. In view of heat transfer augmentation, the structure of the separating and reattaching flow around a surface-mounted obstacle was investigated in detail, together with the corresponding temperature field.

It was shown that the reattachment distance depends on the Reynolds number; the isotherm contours are strongly distorted and pushed down toward the wall due to the back flow in the recirculating zone; the maximum Nusselt number occurs on the upstream side of the reattachment point; and the augmentation effect due to the obstacle is remarkable only in a limited turbulent range of relatively low Reynolds number.
\end{abstract}

\section{Introduction}

To clarify the mechanism of heat transfer augmentation, it is essential to have a detailed understanding of heat transfer in the separated turbulent flow, particularly in the relatively low Reynolds number region. Extensive experimental studies, therefore, have been performed for flow and heat transfer in relation to numerous special geometries such as backward-facing step ${ }^{2,16)}$, surface-mounted obstacle ${ }^{4,8,11,14,15,18)}$, surface with a cavity ${ }^{12)}$ and so on $^{3)}$.

In spite of these numerous experimental studies, the understanding of the phenomena seems to be still insufficient, especially for flow behavior near a surface-mounted obstacle.

In such situations, the use of numerical methods play an important role. According to the authors' knowledge, however, there has been no theoretical investigation of turbulent separated flow except for cases $^{5,9)}$ of a flat plate, a curved surface of very small curvature, and the simplest geometry such as a backward-facing step.

Unfortunately, we have as yet no available turbulent model applicable to cases where the surface is of very large streamwise curvature and the size of the obstacle

\footnotetext{
* Received September 3, 1990. Correspondence concerning this article should be addressed to T. Aragaki.
}

is almost equal to or less than the turbulent boundary layer thickness.

In this study, a simple turbulent model is developed based on the mixing-length hypothesis and is applied to flow problems of complicated geometry by using the Galerkin finite-element method.

The focus here is on two cases as typical examples of turbulent separated flows. The first is a liquid film flowing down the outer wall of a vertical tube, and on the wall is mounted a wire of circular cross-section (like a ring). The second is the flow between two horizontal parallel plates, and a wire or rod of similar geometry is transversely attached to the lower plate.

\section{Theory}

\subsection{Governing equation}

Figures 1(a) and (b) show schematic views of the flows around the surface-mounted obstacle in falling-film and in parallel-plate flow respectively. In the figures, the coordinate systems used are also shown with the domains of calculations.

For an incompressible Newtonian fluid, turbulent flow and heat transfer under steady-state condition are described by the conservation equations of mass, momentum and energy in non-dimensional forms:

$$
\begin{aligned}
& \boldsymbol{\nabla} \cdot \boldsymbol{v}=0 \\
& \operatorname{Rev} \cdot \boldsymbol{\nabla} \boldsymbol{v}=-\boldsymbol{\nabla} \boldsymbol{P}+\boldsymbol{g}+\boldsymbol{\nabla} \cdot[(1+\varepsilon / v) 2 \boldsymbol{S}]
\end{aligned}
$$




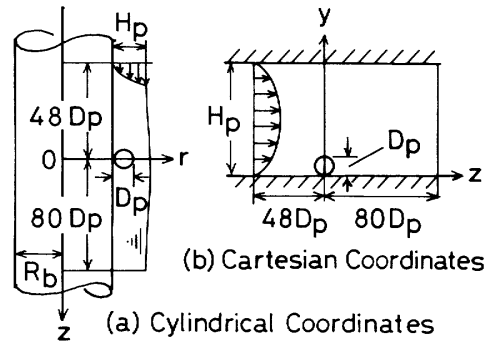

Fig. 1. Schematic view of flow fields and coordinates

$$
\operatorname{Pr} \operatorname{Rev} \cdot \boldsymbol{\nabla} T=\boldsymbol{\nabla} \cdot\left[\left\{1+\left(\operatorname{Pr} / \operatorname{Pr}_{t}\right)(\varepsilon / v)\right\} \boldsymbol{\nabla} T\right]
$$

where $\varepsilon$ is the turbulent eddy viscosity and $S$ is the strain-rate tensor defined by

$$
\boldsymbol{S} \equiv\left[\boldsymbol{\nabla} \boldsymbol{v}+(\boldsymbol{\nabla} \boldsymbol{v})^{T}\right] / 2
$$

In the above equations, dimensionless quantities are defined as

$$
\begin{aligned}
\boldsymbol{v} & \equiv \frac{\boldsymbol{v}^{*}}{V_{a v}} ; \quad P \equiv\left(P^{*}-P_{r e f}^{*}\right) \frac{r_{H}}{\mu V_{a v}} ; \quad T \equiv \frac{T^{*}-T_{0}^{*}}{T_{1}^{*}-T_{0}^{*}} ; \\
\boldsymbol{g} & \equiv \boldsymbol{g}^{*} \frac{r_{H}^{2}}{v V_{a v}} ; \quad R e \equiv \frac{r_{H} V_{a v}}{v}
\end{aligned}
$$

where $V_{a v}$ and $r_{H}$ are the average velocity and the hydraulic depth at the upstream boundary respectively, $P_{r e f}^{*}$ is the reference pressure, and the superscript star $(*)$ denotes dimensional quantity. $P_{r e f}^{*}$ stands for the gas pressure for falling-film and/or an arbitrarily specified pressure for parallel-plate flow. The Reynolds number, $R e$, is defined by $r_{H}$ and $V_{a v}$.

\subsection{Boundary condition}

Film flows: The liquid surface of the falling film is generally perturbed by ripples or waves. However, for simplicity we neglect the existence of ripples or waves on the liquid surface and consider the surface as a time-averaged one. This approximation seems sufficient for the present problems, because the final aim of this study is to gain some knowledge about heat transfer augmentation; in such situation most of the thermal resistance must be unevently distributed in the very vicinity of the solid surface and, consequently, the disturbance due to ripples or waves will have only a minor effect on the heat transfer.

Boundary conditions for film flow can be written in nondimensional forms as follows.

$$
\begin{array}{lll}
\text { (at upstream boundary) } & : & \boldsymbol{v}=V_{i n}(y) \boldsymbol{e}_{z} \\
\text { (on solid surface) } & : & \boldsymbol{v}=0 ; \quad \boldsymbol{v} \cdot \boldsymbol{n}=0 \\
\text { (on liquid surface) } & : & \boldsymbol{v} \cdot \boldsymbol{n}=0 \\
\qquad \boldsymbol{n}-\boldsymbol{n} \cdot(1+\varepsilon / v) 2 \boldsymbol{S} & =-\frac{2 H}{C_{a}} \boldsymbol{n}
\end{array}
$$

The velocity profile $V_{i n}(y)$ at the upstream boundary is assumed to be that for the flow on a smooth vertical tube. The third condition (5c) means that the profile on the liquid surface is unchanged with time (in the sense mentioned above). The last condition, Eq. (5d), is the force balance at the liquid-gas interface ${ }^{1,10)}$, where $C_{a}$ is the capillary number and $\boldsymbol{n}$ the unit normal vector pointing outward at the domain boundary. The mean curvature $H$ of the liquid meniscus can be obtained by the following formula:

$$
2 H=-\nabla_{S} \cdot \boldsymbol{n}
$$

where $\boldsymbol{\nabla}_{S}$ is the surface divergence operator ${ }^{1,19)}$.

Another condition concerns pressure; only for one point on the liquid surface at the downstream boundary is a proper value specified.

The boundary condition for temperature is assumed as

$$
\begin{array}{lll}
\text { (at uptream boundary) } & T=T_{\text {in }} \\
\text { (on solid surface) } & : & T=0 \\
\text { (on liquid surface) } & : & T=1 \\
\text { (on obstacle surface) } & : & \boldsymbol{n} \cdot \boldsymbol{\nabla} T=0
\end{array}
$$

where $T_{i n}$ is the temperature profile at the upstream boundary, for which an analytical solution for the fully developed flow on a smooth vertical tube is used.

Parallel-plate flow: All the boundary conditions mentioned above are again used except those for liquid surface.

\subsection{Turbulent model}

To estimate the eddy viscosity $\varepsilon$ in Eqs. (2) and (3), the simplest method is to use the mixing length hypothesis ${ }^{6}$, which may be written in generalized form $^{17)}$ as

$$
\varepsilon=l^{2}\left[2 S^{*}: S^{*}\right]^{1 / 2}
$$

where $S^{*} \equiv \boldsymbol{S}\left(V_{a v} / r_{H}\right)$ and $l$ is the mixing length. For the inner region of the turbulent boundary layer, the mixing length $l$ is given as proportional to the normal distance $y^{*}$ from the wall:

$$
l=\kappa y^{*} \quad(\kappa=0.4)
$$

For applying the mixing length hypothesis to turbulent flow involving such a complicated wall geometry as those under consideration, the meaning of the distance $y^{*}$ must be modified, because in such a situation the multiple walls (consequently multiple $\left.y^{*}\right)$ will have some effect on the mixing length.

Suppose the nearest two walls as shown in Fig. 2. Let $y_{1}^{*}$ and $y_{2}^{*}$ be the normal distances from the point in question to the corresponding walls, and $y_{1}^{*}$ be smaller than $y_{2}^{*}$. In such a case, the "effective" value of $y^{*}$ will be smaller than $y_{1}^{*}$ due to the influence of the second wall; and if the second wall is very far from the point in question (i.e., $y_{2}^{*} \rightarrow \infty$ ) its effect will vanish.

We therefore simply assume that the effective value of $y^{*}$ is given by the following formula: 

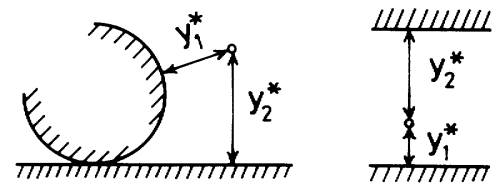

Fig. 2. Normal distance to multiple walls

$$
1 / y^{* n}=1 / y_{1}^{* n}+1 / y_{2}^{* n}
$$

where $n$ is a constant to be decided.

Applying Eq. (9) to the flow in a tube of diameter $2 r_{p}$ and combining with Eq. (8), one gets

$$
l / r_{p}=\kappa \xi\left[1+\{\xi /(2-\xi)\}^{n}\right]^{-1 / n}
$$

where $\xi \equiv y_{1}^{*} / r_{p}$. At the farthest point from the wall (i.e., $\xi=1$ ), Eq. (10) reduces to

$$
l / r_{p}=\kappa 2^{-1 / n} \quad(\text { at } \xi=1)
$$

Figure 3 shows the mixing-length distribution in tube flow for moderate $R e$ numbers according to the measurements of Nikuradse, after Cebeci and Smith ${ }^{6)}$. Based on a possible value of $l / r_{p}($ at $\xi=1)$ from the figure, we can, with the aid of Eq. (11), evaluate the constant $n$. The solid line in the figure is calculated by Eq. (10) with $n=0.72$. In the strict sense, the mixing-length distribution may vary slightly depending on the Reynolds number. For simplicity, however, a single value, $n=0.72$, will be adopted. It is also well known that, very close to the wall, the linear mixing-length relation, Eq. (8), must be modified to account for the wall damping effect ${ }^{6)}$ as

$$
l=\kappa y^{*}\left[1-\exp \left(-\operatorname{Re} u_{\tau} y / A\right)\right]
$$

where $A=26$. For $u_{\tau}$, the dimensionless friction velocity in Eq. (12), we again simply assume that $u_{\tau}=\left(\tau_{s} / \rho\right)^{1 / 2} / V_{a v}$, where $\tau_{s}$ is the wall shear stress at the upstream boundary. After all, Eqs. (9) and (12) will be used, as a first approximation, to evaluate the mixing-length distribution for flows involving complicated geometries, and the validity of the approximation will be examined later.

\section{Numerical Procedure}

The field equations to be solved are discretized by the standard Galerkin finite-element method ${ }^{7,13)}$. In the basic grids used for calculating film flow (parallel-plate flow), there are 1557 (1863) elements, $6523(7747)$ nodes and 14,751 $(17,505)$ freedoms. The element used is the Lagrangian isoparametric quadrilateral. In each finite element, velocity components, $u$ and $w$, and temperature $T$ are approximated by biquadratic shape functions $\phi^{i}$, while pressure $P$ is approximated by bilinear shape functions $\psi^{n}$ as

$$
\begin{aligned}
& u=\phi^{i} u_{i} ; w=\phi^{i} w_{i} ; P=\psi^{n} P_{n}, \quad(i=1 \sim 9, n=1 \sim 4) \\
& T=\phi^{i} T_{i}, \quad(i=1 \sim 9)
\end{aligned}
$$

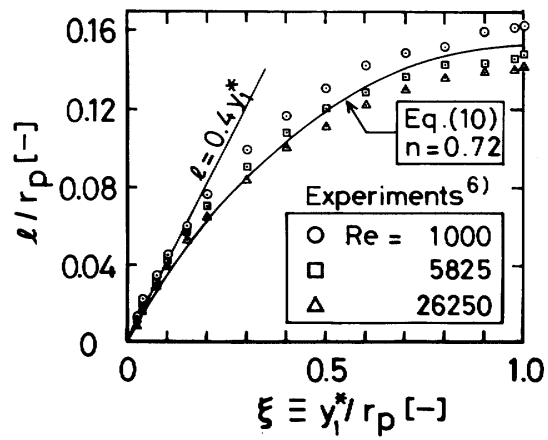

Fig. 3. Mixing-length distribution

where $u$ and $w$ are the radial and axial velocity, respectively, and $u_{i}, w_{i}, P_{n}$ and $T_{i}$ are the nodal values for the corresponding quantities.

According to the standard Galerkin method, the weak formulations of Eqs. (1), (2) and (3) are written as

$$
\begin{gathered}
\int_{D} r^{\alpha} \psi^{m} \boldsymbol{\nabla} \cdot \boldsymbol{v} d D=0 \\
\operatorname{Re} \int_{D} r^{\alpha} \phi^{k} \hat{\boldsymbol{v}} \cdot \boldsymbol{\nabla} \boldsymbol{v} d D-\int_{D} r^{\alpha} \phi^{k} \boldsymbol{g} d D \\
+\alpha \int_{D} \phi^{k}\left(-P+\frac{2 \eta u}{r}\right) d D \boldsymbol{e}_{r}-\int_{D} r^{\alpha} \boldsymbol{\nabla} \phi^{k} \cdot F d D \\
+\int_{S} r^{\alpha} \phi^{k} F \cdot \boldsymbol{n} d S=0 \\
\operatorname{Pr} \operatorname{Re} \int_{D} r^{\alpha} \phi^{k} \boldsymbol{v} \cdot \boldsymbol{\nabla} T d D+\int_{D} r^{\alpha} \boldsymbol{\nabla} \phi^{k} \cdot \zeta \boldsymbol{\nabla} T d D \\
-\int_{S} r^{\alpha} \phi^{k} \zeta \boldsymbol{\nabla} T \cdot \boldsymbol{n} d S=0
\end{gathered}
$$

where $F \equiv P \delta-2 \eta \boldsymbol{S}, \quad \eta \equiv 1+\varepsilon / v, d D \equiv d r d z, \zeta \equiv 1+$ $\left(\operatorname{Pr} / \operatorname{Pr}_{t}\right)(\varepsilon / v)$, and $d S$ is the infinitesimal length along the domain boundary $\partial D$. The parameter $\alpha$ vanishes for plane flow and equals 1 for axisymmetric flow. The velocity, $\hat{v}$, in the first term of Eq. (16) is tentatively treated as a known function in each iteration and assumed to be

$$
\hat{\boldsymbol{v}} \equiv \omega \boldsymbol{v}^{(n)}+(1-\omega) \boldsymbol{v}^{(n-1)}
$$

where $\boldsymbol{v}^{(n)}$ and $\boldsymbol{v}^{(n-1)}$ are the velocities at the $n$ and $(n-1)$ steps respectively in the iteration process, and $\omega$ is a relaxation factor, for which we adopt 0.75 .

Substituting Eq. (13) into Eqs. (15) and (16), we get a set of nonlinear algebraic equations for the flow field:

$$
\left[\begin{array}{lll}
A_{11}^{k i} & A_{12}^{k i} & A_{13}^{k n} \\
A_{21}^{k i} & A_{22}^{k i} & A_{23}^{k n} \\
A_{31}^{m i} & A_{32}^{m i} & 0
\end{array}\right]\left[\begin{array}{l}
u_{i} \\
w_{i} \\
P_{n}
\end{array}\right]=\left[\begin{array}{l}
B_{1}^{k} \\
B_{2}^{k} \\
0
\end{array}\right]
$$

Relations for the temperature field are obtained by combining Eqs. (14) and (17) as

$$
\left[A_{44}^{k i}\right]\left[T_{i}\right]=[0]
$$


All the elements in the above matrices, the definitions of which are standard but lengthy ones and therefore are not described here, must be calculated for each finite element and assembled for all elements, and are then modified by imposing Dirichlet boundary conditions. The equation set resulting from Eq. (18) must then be processed to make the coefficient matrix of much narrower band width; it is simply done by changing the order of unknown variables in solution vector as follows:

$$
\begin{aligned}
& \left(u_{1}, u_{2}, \cdots, w_{1}, w_{2}, \cdots, P_{1}, P_{2}, \cdots\right) \\
& \quad \rightarrow\left(u_{1}, P_{1}, w_{1}, u_{2}, w_{2}, u_{3}, P_{2}, w_{3}, \cdots\right)
\end{aligned}
$$

where the subscript figures denote the current numbers for the whole domain. Consequently, the coefficient matrix and column vector are naturally changed corresponding to the new order of unknown variables; and the resulting equation set with a narrow banded matrix can be easily solved by using a common solver. In this study, a standard Gaussian eliminating method accompanied by normalizing and pivoting routines was adopted.

The shape of the free surface is calculated by the following relation, which is equivalent to $\mathrm{Eq}$. (5c):

$$
d h(z) / d z=u / w
$$

where $h(z)$ is the dimensionless distance of the free surface from the tube wall, and $u$ and $w$ are the radial and axial velocities respectively at the free surface.

By using $h(z)$ and according to Eq. (6), the mean curvature $H$ of the free surface is written as

$$
2 H=\left[h_{z z} / \beta^{2}-1 /(R+h)\right] / \beta
$$

where $R$ is the dimensionless radius of the tube, and $\beta$ is defined by

$$
\beta \equiv\left(1+h_{z}^{2}\right)^{1 / 2}
$$

Iteration for flow calculation is as follows: (i) starting from an initial guess for meniscus shape $h(z)$ and velocity field $\hat{\boldsymbol{v}}$, the domain is divided and the flow calculation is performed based on Eq. (18); (ii) by the update velocity and Eq. (20), the meniscus shape is modified and the velocity field is recalculated; (iii) iteration is repeated until the largest relative change among the components of the solution vector and the meniscus shape becomes less than $10^{-3}$.

The temperature field can be easily determined by using the converged velocity field and Eq. (19).

The scheme for calculating parallel-plate flow is almost the same as that for film flow, and we therefore do not repeat it.

\section{Results and Discussion}

\subsection{Flow field}

Figure 4(a) shows an example of the computed

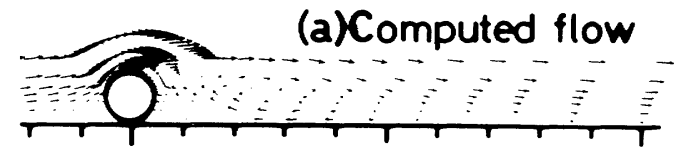

(b) Experiment ${ }^{14)}$
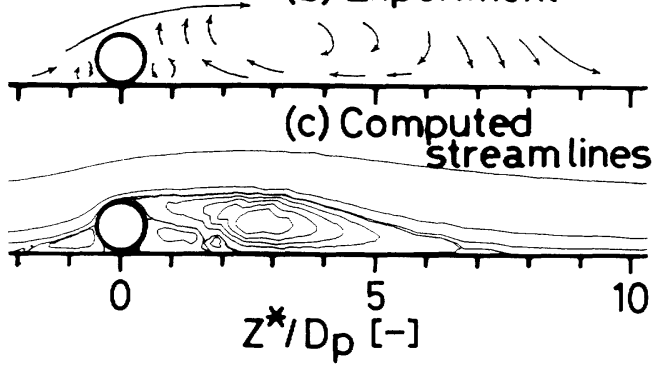

Fig. 4. Comparison of computed results with experiments $^{14)} ; D_{p} / H_{p}=1 / 8 ; R e=4000$

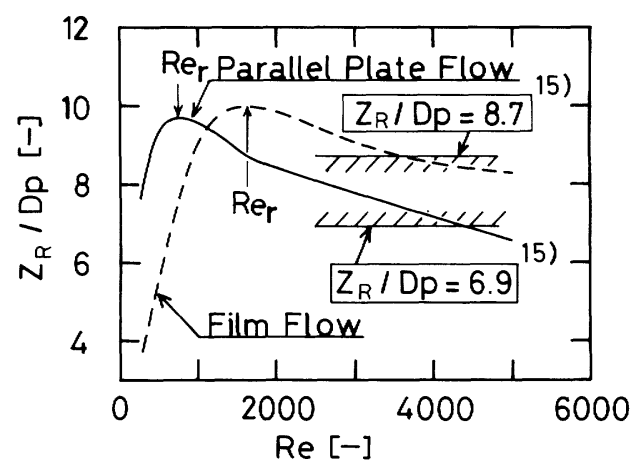

Fig. 5. Comparison of reattachment length with experiments of Miyashita et $a l^{15 \text { ) }}$

velocity field for the parallel plate flow at $R e=4000$, where the width between plates $\left(H_{p}\right)$ is assumed as 8 times of the obstacle diameter $\left(D_{p}\right)$ and $D_{p}=5 \mathrm{~mm}$.

By using flow visualization and a photographic technique, Miyashita et al. ${ }^{14)}$ investigated the flow behavior around a surface-mounted obstacle in a duct flow; Fig. 4(b) shows their result as compared to our theoretical calculation; the correspondence is quite good. In Fig. 4(c), streamline contours are presented to illustrate the flow structure in more detail. It is obvious from the figures that upstream of the obstacle a clockwise recirculating flow occurs, while downstream of the obstacle two recirculating zones of clockwise and counter-clockwise flows are formed.

Figure 5 shows the computed reattachment lengths for parallel-plate flow (solid line) and for film-flow (broken line), where $D_{p}=H_{p} / 8$. The computed lines shown in Fig. 5 are very similar in shape to the measurements by Tropea et al. ${ }^{18)}$ for air flow around a rectangular obstacle, and by Armaly et al. ${ }^{2)}$, for air flow around a backword facing step. On the other hand, Miyashita et al. ${ }^{15)}$ deduced from their visualization experiments that reattachment points exist in a range from $6.9 D_{p}$ to $8.7 D_{p}$ downstream of the obstacle and are almost independent of Reynolds 
number in the fully turbulent region $(R e>2500)$; their result is also shown in Fig. 5 and can be compared with the solid line (parallel-plate flow) because the conditions are almost the same as theirs. We can say that agreement is good, considering the difficulty in experimental determination of reattachment points.

In later discussion, $R e_{r}$, the Reynolds number yielding the maximum reattachment length, will be again referred to in view of heat transfer augmentation.

In Fig. 6, a comparison is made of the computed wall shear stress for parallel-plate flow with the experiments by Miyashita et al. ${ }^{15)}$ for a duct flow, where $\tau_{w}$ and $\tau_{s}$ are the wall shear stresses with and without the obstacle respectively. Inspection of Fig. 6 indicates that the present theory, even based on a simple turbulent model, can predict flow problems, and the agreement is particularly satisfactory at lower Reynolds numbers.

\subsection{Temperature field}

For convenience of inspection, the computed isotherm and streamline contours for film flow are, in parallel, shown in Figs. 7(a) and (b) respectively. It turns out from the figures that the isotherm contours are strongly distorted and pushed down toward the wall due to the back-flow in the recirculating zone.

Based on the temperature field, the local Nusselt number, $N u$, can be determined by $N u=-\boldsymbol{n} \cdot \boldsymbol{\nabla} T$ (at wall).

Figure 8 shows $\mathrm{Nu} / \mathrm{Nu} u_{0}$-distributions downstream of the obstacle for the parallel-plate flows and for the film flows, at different Reynolds numbers, where $N u_{0}$ is the Nusselt number for a smooth surface. Each $N u / N u_{0}$-distribution begins with a locally low value in the very vicinity of the obstacle, while further downstream of the obstacle, $N u / N u_{0}$ increases monotonously with increasing $Z$ and attains a maximum at a location upstream of the reattachment point. A black circle $(0)$ on each line indicates the location of the reattachment point $Z_{R}$. Beyond the maximum, $\mathrm{Nu} / \mathrm{Nu} u_{0}$ decreases monotonously toward 1.0 with increasing $\mathrm{Z}$. It is also obvious from the figure that the effect of the obstacle on the heat transfer rate is strongly dependent on the Reynolds number.

Figure 9 shows the variation of normalized average Nusselt number $\overline{N u} / N u_{0}$, over the range from $Z=0$ to $18 D_{p}$, with $R e$. From the viewpoint of heat transfer augmentaion, the most desirable state, i.e., the maximum $\overline{N u} / N u_{0}$, is attained at about $1.5 R e_{r}$, where $R e_{r}$ is the Reynolds number yielding the maximum reattachment distance $Z_{R}$ as shown in Fig. 5. For $R e$ higher than about $1.5 R e_{r}$, the effect of the obstacle gradually decreases as $R e$ increases.

\section{Conclusion}

A simple model based on the mixing-length

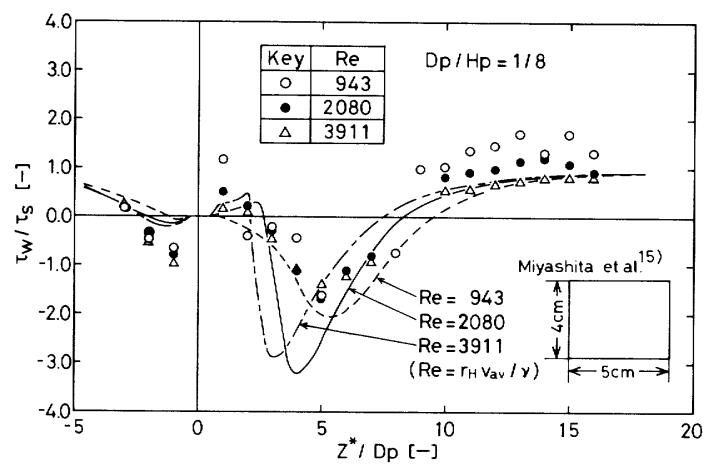

Fig. 6. Comparison of wall shear stress with experiments ${ }^{15}$ )

hypothesis has been developed, and is applied to calculate the turbulent flow and heat transfer around a surface-mounted obstacle, which is of circular cross-section. Calculations were performed for the film flow on the outer wall of a vertical tube and for the flow between parallel plates by using the Galerkin finite-element method.

The structure of the separating and reattaching flow around a surface-mounted obstacle was investigated in detail, together with the corresponding temperature field. It was shown that the reattachment length depends on $R e$; the isotherm contours are strongly distorted and pushed down toward the wall due to the back-flow in the recirculating zone; the location of the maximum Nusselt number is on the upstream side of the reattachment point, and the augmentation effect due to the obstacle is remarkable only in a limited turbulent range of relatively low $R e$.

\section{Acknowledgement}

The authors are grateful to M. Kanda and N. Esaki for their contributions to developing the computer program at the initial stage. This study was partially supported by a grant from Toyoda Physical and Chemical Research Institute. The computations were partially executed on a Fujitsu VP-200 at Nagoya University Computation Center, and partially on a HITAC S-820/80 at the Institute for Molecular Science.

\section{Nomenclature}

$C_{a} \quad=$ Capillary number, $\mu V_{a v} / \sigma \quad[-]$

$D_{p} \quad=$ diameter of obstacle $[\mathrm{m}]$

$\boldsymbol{e}_{r}, \boldsymbol{e}_{z} \quad=$ unit vectors in $r$ - and $z$-directions $\quad[-]$

$g \quad=$ dimensionless gravity; see Eq. (4) [-]

$H \quad=$ dimensionless mean curvature [-]

$H_{p} \quad=$ width between plates or film thickness at upstream boundary

$h(z) \quad=$ dimensionless distance of meniscus from tube wall

$l \quad=$ mixing length $\quad[\mathrm{m}]$

$[-]$

$\boldsymbol{n}=$ unit normal vector pointing outward at boundary

$\mathrm{Nu} \quad=$ Nusselt number

$[-]$

$N u_{0} \quad=$ Nusselt number for smooth surface

$[-]$

$P \quad=$ dimensionless pressure

$[-]$

$P_{g}^{*} \quad=$ gas pressure $\quad[\mathrm{Pa}]$

$P_{\text {ref }}^{*} \quad=$ reference pressure $\quad[\mathrm{Pa}]$ 


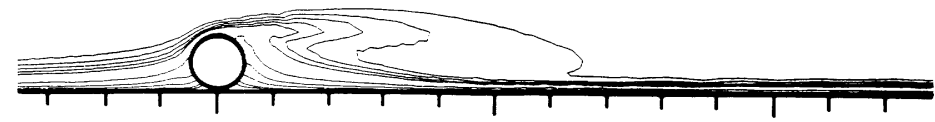

(b) Streamlines

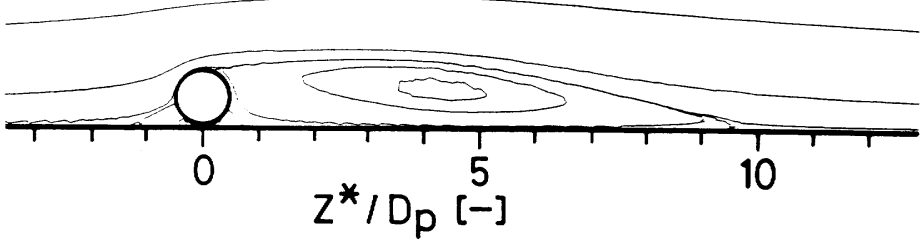

Fig. 7. Computed temperature and flow fields for film flow ( $\left.R e=2500, D_{p} / H_{p}=1 / 8, \operatorname{Pr}=7.0, \operatorname{Pr}_{t}=0.9\right)$

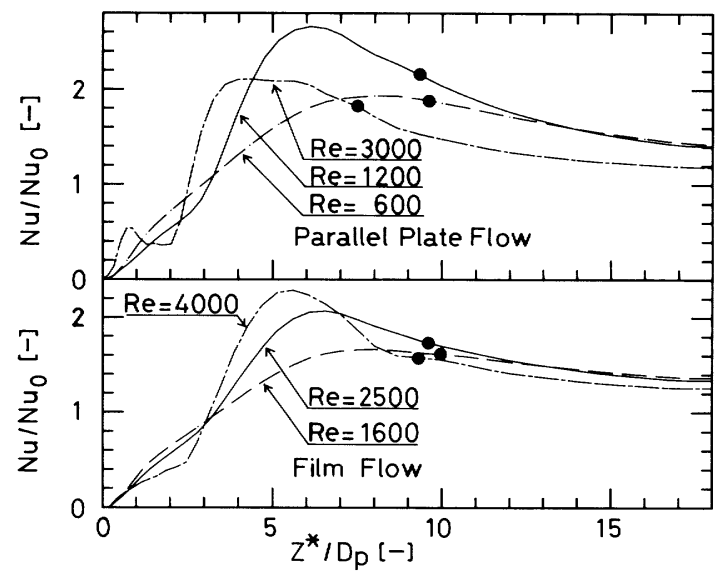

Fig. 8. Local Nusselt number distributions

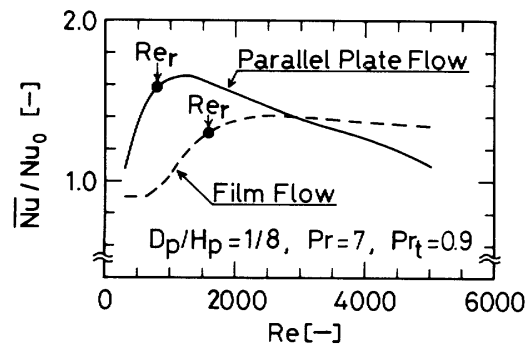

Fig. 9. Average Nusselt number distributions
$=$ Prandtl number, 7.0

$=$ turbulent Prandtl number, 0.9

$=$ dimensionless radial distance, $r^{*} / r_{H}$

$=$ hydraulic depth, used as representative length

$=$ inside radius of tube

$=$ dimensionless radius of tube, $R_{b} / r_{H}$

$=$ outside radius of tube, $9 \times 10^{-3}$

$=$ Reynolds number; see Eq. (4)

$=$ Reynolds number yielding maximum reattachment length

$=$ dimensionless strain rate tensor

$=$ dimensionless temperature, see Eq. (4)

$=$ temperature

$=$ lower temperature at the boundary where obstacle is mounted

\begin{tabular}{|c|c|}
\hline$T_{1}^{*}$ & $\begin{aligned}= & \text { higher temperature at the opposite side } \\
& \text { of } T_{0}^{*} \text {-boundary }\end{aligned}$ \\
\hline$T_{\text {in }}$ & $\begin{array}{l}=\text { dimensionless temperature profile } \\
\text { at upstream boundary }\end{array}$ \\
\hline$u$ & $\begin{aligned}= & \text { dimensionless velocity in } r \text { - or } \\
& y \text {-direction }\end{aligned}$ \\
\hline$u_{\tau}$ & $=$ dimensionless friction velocity \\
\hline$v$ & $=$ dimensionless velocity vector \\
\hline$V_{a v}$ & $\begin{array}{l}=\text { average velocity, used as representative } \\
\text { velocity }\end{array}$ \\
\hline$V_{\text {in }}$ & $\begin{aligned}= & \text { dimensionless velocity profile at upstream } \\
& \text { boundary, } V_{i n}^{*} / V_{a v}\end{aligned}$ \\
\hline$w$ & $=$ dimensionless velocity in $z$-direction \\
\hline$y$ & $=$ dimensionless coordinate; see Fig. 1 \\
\hline$y^{*}$ & $=$ dimensional coordinate, $y r_{H}$ \\
\hline$z$ & $=$ dimensionless coodinate; see Fig. 1 \\
\hline$z_{R}$ & $=$ reattachment length \\
\hline
\end{tabular}

$\langle$ Greek Letters〉

$\begin{array}{ll}\beta & =\text { defined by Eq. (22) } \\ \delta & =\text { unit tensor } \\ \varepsilon & =\text { turbulent eddy viscosity } \\ \kappa & =0.4, \text { von Karman constant } \\ \mu & =\text { viscosity, } 1.254 \times 10^{-3} \\ v & =\text { kinetic viscosity }, 1.202 \times 10^{-6} \\ \rho & =\text { liquid density, } 1.043 \times 10^{3} \\ \tau_{s} & =\text { wall shear stress for smooth surface } \\ \tau_{w} & =\text { wall shear stress } \\ \phi^{i} & =\text { shape function of biquadratic } \\ \psi^{m} & =\text { shape function of bilinear } \\ \omega & =\text { relaxation factor, } 0.75 \\ \langle\text { Superscript }\rangle & \\ * & =\text { denotes dimensional quantity }\end{array}$

\section{Literature Cited}

1) Aragaki, T., K. Murase, H. M. Salah, K. Nagura and S. Toyama: Kagaku Kogaku Ronbunshu, 11, 567 (1985).

2) Armaly, B. F., F. Durst, J. C. F. Pereira and B. Schönung: J. Fluid Mech., 127, 473 (1983).

3) Aung, W. and C. B. Watkins: "Turbulent Forced Convection in Channels and Bundles", edited by S. Kakac and D. B. Spalding, Vol. 1, p. 233, Hemisphere Publishing Co., (1979).

4) Castero, I. P.: J. Fluid Mech., 93, Part 4, 631 (1979).

5) Cebeci, T.: AIAA J. 9, 1868 (1971). 
6) Cebeci, T. and A. M. O. Smith: "Analysis of Turbulent Boundary Layers", p. 111; p. 212, Academic Press (1974).

7) Crochet, M. J. and M. Bezy: J. Non-Newtonian Fluid Mech., 5, 201 (1979).

8) Fujita, H., H. Takahama and H. Eki, JSME, 47, 317 (1981).

9) Goldberg, U. C. and S. R. Chakravarthy: AIAA J., 26, 406 (1988).

10) Higgins, B. G., W. J. Silliman, R. A. Brown and L. E. Scriven: Ind. Eng. Chem. Fundam., 16, No. 4, 393 (1977).

11) Hijikata, K., Y. Mori and H. Ishiguro: JSME, 50, 2555 (1984).

12) Hiwada, M., I. Mabuchi and M. Kumada, JSME, 49, 1895 (1983).

13) Huebner, K. H.: "The Finite Element Method for Engineers",
John Wiley \& Sons (1975).

14) Miyashita, H., Y. Shiomi and K. Wakabayashi: Kagaku Kogaku Ronbunshu, 7, 81 (1981).

15) Miyashita, H., A. Takayanagi and K. Wakabayashi: Kagaku Kogaku Ronbunshu, 6, 152 (1980).

16) Nezu, I and H. Nakagawa: "Turbulent Shear Flows 6", p. 313, Springer-Verlag, Berlin and Heidelberg (1989).

17) Tanahashi, T.: "Renzokutai no Rikigaku (3)", p. 118, Riko Tosyo (1986).

18) Tropea, C. D. and R. Gackstatter: J. Fluid Engng., 107, 489 (1985).

19) Weatherburn, C. E.: "Differential Geometry of Three Dimensions", p. 225, Cambridge Univ. Press (1974). 